\title{
Measuring the ISW effect with next-generation radio surveys
}

\author{
Mario Ballardini ${ }^{1,2}$ *, Roy Maartens ${ }^{1,3} \dagger$ \\ ${ }^{1}$ Department of Physics \& Astronomy, University of the Western Cape, Cape Town 7535, South Africa \\ ${ }^{2}$ INAF/OAS Bologna, via Gobetti 101, I-40129 Bologna, Italy \\ ${ }^{3}$ Institute of Cosmology \& Gravitation, University of Portsmouth, Portsmouth PO1 3FX, UK
}

14 February 2019

\begin{abstract}
The late-time integrated Sachs-Wolfe (ISW) signal in the CMB temperature anisotropies is an important probe of dark energy when it can be detected by crosscorrelation with large-scale structure surveys. Because of their huge sky area, surveys in the radio are well-suited to ISW detection. We show that $21 \mathrm{~cm}$ intensity mapping and radio continuum surveys with the SKA in Phase 1 promise a $\sim 5 \sigma$ detection if we use tomography, with a similar forecast for the precursor EMU survey. In SKA Phase 2 , the $21 \mathrm{~cm}$ galaxy redshift survey and the continuum survey could deliver a $\sim 6 \sigma$ detection. Our analysis of the radio surveys aims for theoretical accuracy on large scales. Firstly, we include all the effects on the radio surveys from observing on the past lightcone: redshift-space distortions and lensing magnification can have a significant impact on the ISW signal to noise ratio, while Doppler and other relativistic distortions are not significant. Secondly, we use the full information in the observable galaxy angular power spectra $C_{\ell}\left(z, z^{\prime}\right)$, by avoiding the Limber approximation and by including all cross-correlations between redshift bins in the covariance. Without these cross-bin correlations, the ISW signal to noise ratio is biased.
\end{abstract}

Key words: cosmology: observations - cosmic background radiation - large-scale structure of Universe.

\section{INTRODUCTION}

The late-time integrated Sachs-Wolfe (ISW) effect in the cosmic microwave background (CMB) arises from the time variation of the gravitational potentials along the line of sight (Sachs \& Wolfe 1967). CMB photons are gravitational redshifted while travelling through potential wells and hills connected to matter over- and under-densities. In a matterdominated universe the local gravitational potentials are constant and the net effect of a photon falling into a gravity well and coming out is zero. By contrast, gravitational potentials decay during the $\Lambda$-dominated phase, leading to a net change in photon temperature and in the observed CMB temperature anisotropies. These potential fluctuations are induced by density perturbations at relatively low redshifts and generate a non-vanishing cross-correlation component between CMB temperature anisotropies and CMB lensing, and between $\mathrm{CMB}$ temperature anisotropies and number count fluctuations.

Many measurements of the ISW signal based on cross-

* E-mail: mario.ballardini@gmail.com

† E-mail: roy.maartens@gmail.com correlating the CMB with large-scale structure tracers have been performed (Crittenden \& Turok 1996; Nolta et al. 2004; Fosalba \& Gaztanaga 2004; Boughn \& Crittenden 2004; Afshordi et al. 2004; Vielva et al. 2006; McEwen et al. 2007; Ho et al. 2008; Giannantonio et al. 2008; Sarkar et al. 2009; Hernandez-Monteagudo 2010; Taburet et al. 2011; Ilic et al. 2011; Schiavon et al. 2012; Barreiro et al. 2013; Ade et al. 2014; Ferraro et al. 2015; Manzotti \& Dodelson 2014; Ade et al. 2016; Shajib \& Wright 2016; Bianchini et al. 2016; Stölzner et al. 2018; Maniyar et al. 2018). Measurements have used different matter tracers: radio source catalogues, spectroscopic and photometric galaxy surveys, photometric quasars, thermal Sunyaev-Zeldovich, cosmic infrared background, and CMB lensing. Alternatively, the stacking of CMB data in correspondence with superclusters and supervoids has also been used for ISW detection (Granett et al. 2008; Papai et al. 2011; Ilic et al. 2011; Ade et al. 2014, 2016).

ISW fluctuations contribute mainly to large angular scales, $\ell \lesssim 100$, of the CMB temperature angular power spectrum, since there is little power in the potentials at late times on scales that entered the Hubble radius during radiation domination (Kofman \& Starobinsky 1985). For this 
reason, wide surveys are optimal to tackle ISW detection. Such surveys can probe the large-scale structure on ultralarge (super-equality) scales, facilitating measurements of not only the ISW, but also primordial non-Gaussianity, the primordial power spectrum and relativistic observational effects on number counts and intensity (Raccanelli et al. 2012; Maartens et al. 2013; Raccanelli et al. 2015; Camera et al. 2015; Raccanelli et al. 2016; Alonso et al. 2015; Fonseca et al. 2015; Ballardini et al. 2018; Karagiannis et al. 2018; Ballardini 2019; Bernal et al. 2018). Among the nextgeneration surveys of the large-scale structure, radio surveys promise to deliver the largest volumes, using neutral hydrogen (HI) $21 \mathrm{~cm}$ emission or radio continuum emission of galaxies (Maartens et al. 2015). Radio surveys can maximise the synergies with CMB maps, thanks to their large overlapping sky area.

In this paper, we test the feasibility of detecting the ISW signal with future cosmological surveys in Phase 1 of the Square Kilometre Array (SKA) (Bacon et al. 2018), together with two of its precursor surveys, MeerKLASS (Santos et al. 2017) and EMU (Norris et al. 2011). We also consider the more futuristic 'billion galaxy' spectroscopic survey and continuum survey in SKA Phase 2.

We begin by quantifying the theoretical signal-to-noise ratio (SNR) for ISW detection through angular cross-power spectra of CMB temperature and number count/ intensity. Then we simulate ISW and large-scale structure (LSS) maps to identify the quality of the reconstruction for the radio surveys considered. We explore the impact of lensing magnification and other relativistic effects in LSS maps on the crosscorrelation with CMB temperature maps (see also LoVerde et al. 2007; Challinor \& Lewis 2011; Renk et al. 2016). When these effects are not modelled, we use the incorrect theoretical model, potentially leading to a bias in the ISW reconstruction. In addition, we highlight the importance of the cross-correlations between redshift bins when a tomographic approach is used. Without these cross-bin correlations, the covariance is not correctly modelled, leading to a bias in the ISW signal to noise.

The paper is organized as follows. We review the angular cross-power spectrum in section 2, and the SNR calculation in section 3 . In section 4 , we describe the properties of the surveys. Section 5 discusses our results for the SNR analysis. Our procedure for ISW reconstruction is described in section 6 , together with discussion on the accuracy of using the average correlation coefficient and on map residuals between the true ISW map and the reconstructed one in pixel space. We draw our conclusions in section 7 .

\section{CMB-LSS CROSS-CORRELATION}

We study the cross-correlation between the LSS angular power spectrum derived in linear perturbation theory assuming general relativity (GR) and the ISW contribution to the CMB temperature angular power spectrum. There is a simple way to relate number count results to intensity mapping, which we describe below. Therefore in this section we consider the observed number counts for a magnitudelimited survey.

In Newtonian gauge and in Fourier space, the multipoles of the observed number counts can be split into a standard term, which includes RSD by convention, and the GR contributions which are effectively the corrections to the standard Newtonian approximation:

$\Delta_{\ell}(\boldsymbol{k}, z)=\Delta_{\ell}^{\mathrm{N}}(\boldsymbol{k}, z)+\Delta_{\ell}^{\mathrm{GR}}(\boldsymbol{k}, z)$.

The Newtonian term is the number density contrast plus the standard RSD term:

$\Delta_{\ell}^{\mathrm{N}}=b \delta_{\boldsymbol{k}}^{\mathrm{c}} j_{\ell}(k \chi)+\frac{k v_{\boldsymbol{k}}}{\mathcal{H}} j_{\ell}^{\prime \prime}(k \chi)$.

Here $\delta_{\boldsymbol{k}}^{\mathrm{c}}(z)$ is the comoving matter density contrast, used in order to impose a physical model of scale-independent bias $b(z)$ (Challinor \& Lewis 2011; Bruni et al. 2012; Jeong et al. 2012; Baldauf et al. 2012). The peculiar velocity of the source is $\boldsymbol{v}$, with $v_{\boldsymbol{k}}(z)=\left|\boldsymbol{v}_{\boldsymbol{k}}(z)\right|$, and $j_{\ell}$ are spherical Bessel functions, where prime denotes $\mathrm{d} / \mathrm{d}(k \chi) \cdot \mathcal{H}(z)=(1+$ $z)^{-1} H(z)$ is the conformal Hubble parameter and $\chi(z)$ is the comoving distance.

The GR corrections to $\Delta^{\mathrm{N}}$ are given by (Challinor \& Lewis 2011):

- the lensing convergence contribution $(\mathrm{L})$, which is $\propto \delta_{k}^{\mathrm{c}}$;

- the Doppler effect due to redshift perturbations from peculiar velocity $(\mathrm{V})$, which is $\propto(\mathcal{H} / k) \delta_{\boldsymbol{k}}^{\mathrm{c}}$;

- ultra-large scale terms (ULS), which are $\propto(\mathcal{H} / k)^{2} \delta_{\boldsymbol{k}}^{\mathrm{c}}$, and come from the gravitational potentials:

$\mathrm{d} s^{2}=\left[-(1+2 \psi) \mathrm{d} \eta^{2}+(1-2 \phi) \mathrm{d} \boldsymbol{x}^{2}\right]$.

In detail (Fonseca et al. 2015):

$\Delta_{\ell}^{\mathrm{GR}} \equiv \Delta_{\ell}^{\mathrm{L}}+\Delta_{\ell}^{\mathrm{V}}+\Delta_{\ell}^{\mathrm{ULS}}$,

$\Delta_{\ell}^{\mathrm{L}}=\frac{\ell(\ell+1)}{2}(2-5 s)$

$\times \int_{0}^{\chi} \mathrm{d} \tilde{\chi} \frac{(\chi-\tilde{\chi})}{\chi \tilde{\chi}}\left[\psi_{\boldsymbol{k}}(\tilde{\chi})+\phi_{\boldsymbol{k}}(\tilde{\chi})\right] j_{\ell}(k \tilde{\chi})$,

$\Delta_{\ell}^{\mathrm{V}}=\left[\frac{2-5 s}{\mathcal{H} \chi}+5 s-b_{e}+\frac{\dot{\mathcal{H}}}{\mathcal{H}^{2}}\right] v_{\boldsymbol{k}} j_{\ell}^{\prime}(k \chi)$,

where

$s\left(z, m_{*}\right)=\frac{\partial \log \overline{\mathcal{N}}\left(z, m<m_{*}\right)}{\partial m_{*}}$,

$b_{e}\left(z, m_{*}\right)=-\frac{\partial \ln \left[(1+z)^{-3} \overline{\mathcal{N}}\left(z, m<m_{*}\right)\right]}{\partial \ln (1+z)}$,

are the magnification and evolution bias, and $\overline{\mathcal{N}}$ is the background number density of sources with luminosity above the threshold.

The ULS contribution is made up of local and integrated terms:

$$
\begin{aligned}
\Delta_{\ell}^{\mathrm{ULS}}= & \left\{\left[\frac{2-5 s}{\mathcal{H} \chi}+5 s-b_{e}+\frac{\dot{\mathcal{H}}}{\mathcal{H}^{2}}+1\right] \psi_{\boldsymbol{k}}+\right. \\
& \left.(5 s-2) \phi_{\boldsymbol{k}}+\frac{\dot{\phi}_{\boldsymbol{k}}}{\mathcal{H}}+\left(b_{e}-3\right) \mathcal{H} \frac{v_{\boldsymbol{k}}}{k}\right\} j_{\ell}(k \chi)+ \\
& {\left[\frac{2-5 s}{\mathcal{H} \chi}+5 s-b_{e}+\frac{\dot{\mathcal{H}}}{\mathcal{H}^{2}}\right] \int_{0}^{\chi} \mathrm{d} \tilde{\chi}\left[\dot{\psi}_{\boldsymbol{k}}(\tilde{\chi})+\dot{\phi}_{\boldsymbol{k}}(\tilde{\chi})\right] j_{\ell}(k \tilde{\chi}) } \\
& +\frac{(2-5 s)}{\chi} \int_{0}^{\chi} \mathrm{d} \tilde{\chi}\left[\psi_{\boldsymbol{k}}(\tilde{\chi})+\phi_{\boldsymbol{k}}(\tilde{\chi})\right] j_{\ell}(k \tilde{\chi}) .
\end{aligned}
$$

The first two lines of (9) are local Sachs-Wolfe type terms. The velocity term $v_{\boldsymbol{k}} / k$ - which arises from expressing the Newtonian-gauge number density contrast in comoving gauge - can be rewritten as a potential term using the Poisson and continuity equations (Fonseca et al. 2018). The last 
two lines are nonlocal integrated terms, from the ISW and time-delay effects in the LSS density contrast.

The angular power spectra of the LSS density contrast and the CMB ISW are (suppressing the redshift dependence):

$C_{\ell}^{\mathrm{XY}}=4 \pi \int \frac{\mathrm{d} k}{k} \mathcal{P}_{\mathcal{R}}(k) I_{\ell}^{\mathrm{X}}(k) I_{\ell}^{\mathrm{Y}}(k)$,

where $\mathrm{X}, \mathrm{Y}=\Delta$ or ISW and we use the convention $C_{\ell}^{\mathrm{XX}}=$ $C_{\ell}^{\mathrm{X}}$. In (10), $\mathcal{P}_{\mathcal{R}}$ is the dimensionless primordial power spectrum and the kernels are:

$$
\begin{aligned}
I_{\ell}^{\Delta}\left(k, z_{\mathrm{i}}\right) & =\int \mathrm{d} z W\left(z, z_{\mathrm{i}}\right) \Delta_{\ell}(k, z), \\
I_{\ell}^{\mathrm{ISW}}(k) & =\int \mathrm{d} z e^{-\tau(z)} \mathcal{T}_{\dot{\phi}+\dot{\psi}}(k, z) j_{\ell}(k \chi(z)) .
\end{aligned}
$$

Here $W\left(z, z_{\mathrm{i}}\right)$ is the window function for the LSS redshift bin $z_{\mathrm{i}} \pm \Delta z / 2, \tau$ is the optical depth, and $\Delta_{\ell}(k, z)$ is obtained from (2), (5), (6), (9) through replacing $\delta_{\boldsymbol{k}}^{\mathrm{c}}(z)$ by its transfer function $\mathcal{T}_{\delta^{c}}(k, z)$, and similarly for $v_{\boldsymbol{k}}, \psi_{\boldsymbol{k}}, \phi_{\boldsymbol{k}}$. We use top-hat windows for the HI surveys (which have excellent spectroscopic accuracy), and Gaussian windows for the continuum survey. We do not use the Limber approximation, which is not accurate on the large scales where the ISW signal is strongest.

\section{THEORETICAL SIGNAL-TO-NOISE}

The observed LSS auto-power spectra are

$\bar{C}_{\ell}^{\Delta}\left(z_{\mathrm{i}}\right)=C_{\ell}^{\Delta}\left(z_{\mathrm{i}}\right)+\mathcal{N}_{\ell}\left(z_{\mathrm{i}}\right)$,

where $\mathcal{N}_{\ell}$ is shot noise for galaxies and thermal noise for intensity mapping (see below).

In order to quantify the possibility of extracting the ISW signal from the CMB, we follow (Cooray 2002; Afshordi 2004) and use the signal-to-noise ratio (SNR) defined by:

$$
\left(\frac{S}{N}\right)^{2}=\sum_{\ell=2}^{\ell_{\max }}\left(\mathbf{C}_{\ell}^{\Delta \mathrm{ISW}}\right)^{\dagger}\left(\mathbf{C}_{\ell}^{\mathrm{cov}}\right)^{-1} \mathbf{C}_{\ell}^{\Delta \mathrm{ISW}}
$$

Here $\mathbf{C}_{\ell}^{\Delta \mathrm{ISW}}\left(z_{\mathrm{i}}\right)$ is the vector of the angular cross-power spectra, and the covariance matrix elements are:

$\mathbf{C}_{\ell}^{\mathrm{cov}}\left(z_{\mathrm{i}}, z_{\mathrm{j}}\right)=\frac{C_{\ell}^{\Delta \mathrm{ISW}}\left(z_{\mathrm{i}}\right) C_{\ell}^{\Delta \mathrm{ISW}}\left(z_{\mathrm{j}}\right)+\bar{C}_{\ell}^{\Delta}\left(z_{\mathrm{i}}, z_{\mathrm{j}}\right) \bar{C}_{\ell}^{\mathrm{ISW}}}{(2 \ell+1) f_{\mathrm{sky}}}$

where $f_{\text {sky }}$ is the common sky fraction of the LSS and CMB surveys. Cross-bin LSS correlations $\bar{C}_{\ell}^{\Delta}\left(z_{\mathrm{i}}, z_{\mathrm{j}}\right), \mathrm{i} \neq \mathrm{j}$, do not enter the ISW signal $\mathbf{C}_{\ell}^{\Delta \text { ISW }}$, but they do affect the SNR, via the covariance matrix (15). Neglecting these cross-bin correlations can therefore bias the SNR.

For the ISW contribution to the CMB, the primary temperature auto-power is part of the noise:

$\bar{C}_{\ell}^{\mathrm{ISW}}=C_{\ell}^{\mathrm{ISW}}+C_{\ell}^{\mathrm{TT}}+\mathcal{N}_{\ell}^{\mathrm{T}}$.

At low and intermediate multipoles, where the ISW signal is not suppressed, the instrumental noise on the CMB temperature signal can be neglected, i.e. $\mathcal{N}_{\ell}^{\mathrm{T}} \approx 0$.

The CMB E-mode polarization can be used to improve the ISW signal in the CMB temperature angular power spectrum, thanks to the TE correlation between the two spectra.
By including E-mode polarization information, it is possible to decrease the effective cosmic variance on the CMB temperature and ISW angular power spectra:

$\bar{C}_{\ell}^{\mathrm{ISW}}=C_{\ell}^{\mathrm{ISW}}+C_{\ell}^{\mathrm{TT}}-\frac{\left(C_{\ell}^{\mathrm{TE}}\right)^{2}}{C_{\ell}^{\mathrm{EE}}}+\mathcal{N}_{\ell}^{\mathrm{T}}$.

The inclusion of CMB polarization in the analysis increases the SNR by $\sim 18 \%$ (Frommert \& Ensslin 2008; Giannantonio et al. 2012; Ballardini et al. 2019). Therefore in the SNR calculation we always include the E-mode polarization information according to (17).

\section{RADIO SURVEY SPECIFICATIONS}

In this section we provide the LSS survey details used for the analysis.

\subsection{HI intensity mapping survey}

HI intensity mapping (IM) surveys do not attempt to detect individual HI galaxies, but measure the total signal in each pixel to produce maps of the large-scale fluctuations in HI galaxy clustering (with very accurate redshifts) (Battye et al. 2004; Wyithe \& Loeb 2008; Chang et al. 2008; Bull et al. 2015; Santos et al. 2015; Kovetz et al. 2017). The flux density measured is converted into an effective brightness temperature of the $21 \mathrm{~cm}$ emission:

$T_{\mathrm{HI}}=\bar{T}_{\mathrm{HI}}\left(1+\delta_{\mathrm{HI}}\right) \mu \mathrm{K}$.

$\mathrm{HI}$ is expected to be a biased tracer of the cold dark matter distribution, just as galaxies are, because the HI content of the Universe is expected to be localized within galaxies after reionization. We use the fitting formulas (Santos et al. 2017):

$b_{\mathrm{HI}}(z)=\frac{b_{\mathrm{HI}}(0)}{0.677105}\left[0.66655+0.17765 z+0.050223 z^{2}\right]$,

$\bar{T}_{\mathrm{HI}}(z)=0.055919+0.23242 z-0.024136 z^{2} \mathrm{mK}$,

where $\Omega_{\mathrm{HI}}(0) b_{\mathrm{HI}}(0)=4.3 \times 10^{-4}$ and $\Omega_{\mathrm{HI}}(0)=4.86 \times 10^{-4}$.

The observed brightness temperature contrast may be obtained from the number count case by using effective values for the evolution and magnification biases as follows (Hall et al. 2013; Fonseca et al. 2018):

$b_{e \mathrm{HI}}(z)=-\frac{\partial \ln \left[(1+z)^{-1} \mathcal{H}(z) \bar{T}_{\mathrm{HI}}(z)\right]}{\partial \ln (1+z)}, \quad s_{\mathrm{HI}}=\frac{2}{5}$.

Note that the lensing magnification contribution is thus zero at first order.

We consider IM in single-dish mode, i.e. adding up all dishes independently as opposed to combining them via interferometry, using SKA1-MID Band 1 and the proposed IM survey MeerKLASS on the precursor MeerKAT. Single-dish mode is the most efficient way to probe cosmological scales with IM (Santos et al. 2015). Assuming scale-independence and no correlation between the noise in different frequency channels, the noise variance per steradian in the $i$-frequency 
channel is (Knox 1995; Bull et al. 2015):

$$
\begin{aligned}
\mathcal{N}_{\ell}^{\mathrm{HI}}\left(\nu_{\mathrm{i}}\right) & =\frac{4 \pi f_{\text {sky }} T_{\text {sys }}^{2}\left(\nu_{\mathrm{i}}\right)}{2 N_{\mathrm{dish}} t_{\text {tot }} \Delta \nu}, \\
T_{\text {sys }}(\nu) & =25+60\left(\frac{300 \mathrm{MHz}}{\nu}\right)^{2.55} \mathrm{~K} .
\end{aligned}
$$

For MeerKLASS, we assume $N_{\text {dish }}=64, t_{\text {tot }}=4,000$ hr over $4,000 \operatorname{deg}^{2}\left(f_{\text {sky }} \simeq 0.1\right)$ in the redshift ranges $0 \leq$ $z \leq 0.58(1670 \geq \nu \geq 900 \mathrm{MHz}, \mathrm{L}$ Band $)$ and $0.4 \leq z \leq 1.45$ (1015 $\geq \nu \geq 580 \mathrm{MHz}$, UHF Band) (Santos et al. 2017).

For SKA1-MID, we assume $N_{\text {dish }}=197, t_{\text {tot }}=10^{4} \mathrm{hr}$ observing over $20,000 \operatorname{deg}^{2}\left(f_{\text {sky }} \simeq 0.5\right)$ in the redshift range $0.35 \leq z \leq 3.05(1050 \geq \nu \geq 350 \mathrm{MHz}$, Band 1) (Bacon et al. 2018).

\subsection{Radio continuum survey}

A continuum survey detects the total radio emission of galaxies, which is dominated by synchrotron radiation. As a consequence, there is no redshift information, and redshifts must be obtained by cross-matching with optical/ infrared surveys (or HI IM surveys). On the other hand, radio galaxies are detected out to very high redshift.

For a continuum galaxy survey with SKA1-MID, we assume the same frequency band and sky area as for the IM survey, with a source detection limit $S_{\text {cut }}=22 \mu \mathrm{Jy}$ (Bacon et al. 2018). We study also an optimistic case for SKA1MID with $S_{\text {cut }}=10 \mu \mathrm{Jy}$. For SKA2 we assume $30,000 \mathrm{deg}^{2}$ $\left(f_{\mathrm{sky}} \simeq 0.7\right)$ and $S_{\mathrm{cut}}=1 \mu \mathrm{Jy}$.

As an SKA precursor continuum galaxy survey, we consider EMU on ASKAP (Norris et al. 2011; Bernal et al. 2018), covering the frequency range $700-1450 \mathrm{MHz}$, with $f_{\text {sky }} \simeq 0.7$ and $S_{\text {cut }}=100 \mu \mathrm{Jy}$.

The redshift distribution, bias, magnification bias and evolution bias are predicted using the publicly available code $^{1}$ developed by Alonso et al. (2015), which provides semi-analytical fits based on the simulations described in Wilman et al. (2008).

\subsection{HI galaxy survey}

The models for the number counts and the clustering, evolution and magnification biases of the HI galaxy distribution are given in Camera et al. (2015), for various flux thresholds.

For SKA1, the sky area and redshift coverage are too low for detecting the ISW. SKA2 specifications have not been formalised, but the rms noise is expected to be $\sim 10$ times smaller than SKA1, and the sky coverage increased from 20,000 to $30,000 \mathrm{deg}^{2}\left(f_{\text {sky }} \simeq 0.7\right)$. We assume a total observation time of $10^{4} \mathrm{hr}$ in a redshift range $0.1 \leq z \leq 2$, and with flux threshold $1 \mu \mathrm{Jy}$. This is the so-called 'billion galaxy' survey.

Note that plots of the number density, clustering bias, evolution bias, and magnification bias as functions of $z$ for the three types of radio survey can be found in Camera et al. (2015) and Alonso et al. (2015).

${ }^{1}$ http://intensitymapping.physics.ox.ac.uk/codes.html

\section{RESULTS}

\subsection{HI IM survey}

Table 1 summarises the SNR obtained by correlating the observed HI brightness temperature contrast $\Delta_{\mathrm{HI}}$ with the CMB ISW signal. We calculate the SNR in two different configurations, for MeerKAT L-band/ UHF-band/ SKA1MID Band 1 respectively:

$$
\begin{aligned}
& 1 z \text {-bin with edges }[0,0.58] /[0.4,1.45] /[0.35,3.05] \\
& 5 / 11 / 27 z \text {-bins with } \Delta z=0.1
\end{aligned}
$$

In each case, we compare the SNR using the Newtonian approximation $\Delta^{\mathrm{N}}$ with the SNR using the full GR result $\Delta=\Delta^{\mathrm{N}}+\Delta^{\mathrm{GR}}$. For the cases with tomography, we also show the effect (in brackets) of neglecting cross-bin correlations in the brightness temperature.

The SNR for MeerKLASS IM surveys is strongly limited by the survey area of $4,000 \mathrm{deg}^{2}$. Moreover, the instrumental noise limits the possibility to increase the SNR by considering many slices in redshift. We find a SNR $\sim 1.1-1.3$ for the L-band and $\sim 1.7-1.9$ for the UHF-band. For an SKA1-MID Band 1 IM survey, we find a SNR of $\sim 3.7$ using the whole survey as one single redshift bin, increasing up to $\sim 4.7$ using tomography.

In IM, the lensing magnification contribution $\Delta^{\mathrm{L}}$ given by (5) is zero, as follows from (21). As a further consequence of $s_{\mathrm{HI}}=2 / 5$, parts the other GR terms, $\Delta^{\mathrm{V}}$ (Doppler) given by (6) and $\Delta^{\mathrm{ULS}}$ (potentials) given by (9), are suppressed. The contribution $\Delta^{\text {GR }}$ from the total GR correction in the observed temperature contrast $\Delta$ is much smaller than the Newtonian $\Delta^{\mathrm{N}}$ contribution, even at high redshift. This can be seen in figure 1. Table 1 shows that for IM there is a negligible difference between the SNR in full GR and the SNR in the Newtonian approximation.

In all cases, tomography improves the $S N R$. If we neglect the IM cross-bin correlations in the covariance (15), then the SNR is biased downward (numbers in brackets) - i.e. cross-bin correlations increase the SNR. Neglecting cross-bin correlations in IM leads to a false reduction in SNR, due to incorrect modelling of the covariance.

In Pourtsidou et al. (2017), the SNR for ISW detection is calculated for MeerKLASS UHF-band and SKA1MID Band 1, assuming independent redshift bins with a width of $\Delta z=0.1$. They therefore neglect cross-bin correlations, and they find a SNR of 1.5 and 4.6. The difference with our results is likely due to their assumptions of a different best-fit cosmology, a simplified redshift independent bias, $b_{\mathrm{HI}}(z)=1$, and (for SKA1) a larger sky coverage of $30,000 \mathrm{deg}^{2}$.

\subsection{Radio continuum survey}

In table 2, we show the SNR obtained by correlating the observed number count contrast with the CMB ISW, for EMU and SKA1 continuum surveys. We use the binning configurations:

$$
\begin{aligned}
& 1 z \text {-bin with edges }[0,5] \\
& 5 z \text {-bins with edges }[0,0.5,1,1.5,2,5]
\end{aligned}
$$

The second configuration applies only to SKA1 and is given in Bacon et al. (2018), based on the argument that suffi- 
Table 1. SNR for ISW detection from IM surveys with MeerKAT and SKA1-MID, using binning configurations (24) and (25). SNR is calculated using the full HI temperature contrast $\Delta^{\mathrm{N}}+\Delta^{\mathrm{GR}}$ given by (1) (left column) and its Newtonian approximation $\Delta^{\mathrm{N}}$ given by (2) (right column). Numbers in round brackets indicate neglect of the contribution from cross-bin correlations in (14).

\begin{tabular}{ccccccc}
\hline & \multicolumn{2}{c}{$\begin{array}{c}\text { MeerKLASS } \\
\text { L-band }\end{array}$} & \multicolumn{2}{c}{$\begin{array}{c}\text { MeerKLASS } \\
\text { UHF-band }\end{array}$} & \multicolumn{2}{c}{$\begin{array}{c}\text { SKA1-MID } \\
\text { Band 1 }\end{array}$} \\
\hline & $\Delta$-ISW & $\Delta^{\mathrm{N}}$-ISW & $\Delta$-ISW & $\Delta^{\mathrm{N}}$-ISW & $\Delta$-ISW & $\Delta^{\mathrm{N}}$-ISW \\
\hline 1 bin & 1.1 & 1.1 & 1.7 & 1.7 & 3.7 & 3.7 \\
$\Delta z=0.1$ bins & $1.3(1.2)$ & $1.3(1.2)$ & $1.9(1.6)$ & $1.9(1.6)$ & $4.7(3.9)$ & $4.7(3.9)$ \\
\hline
\end{tabular}
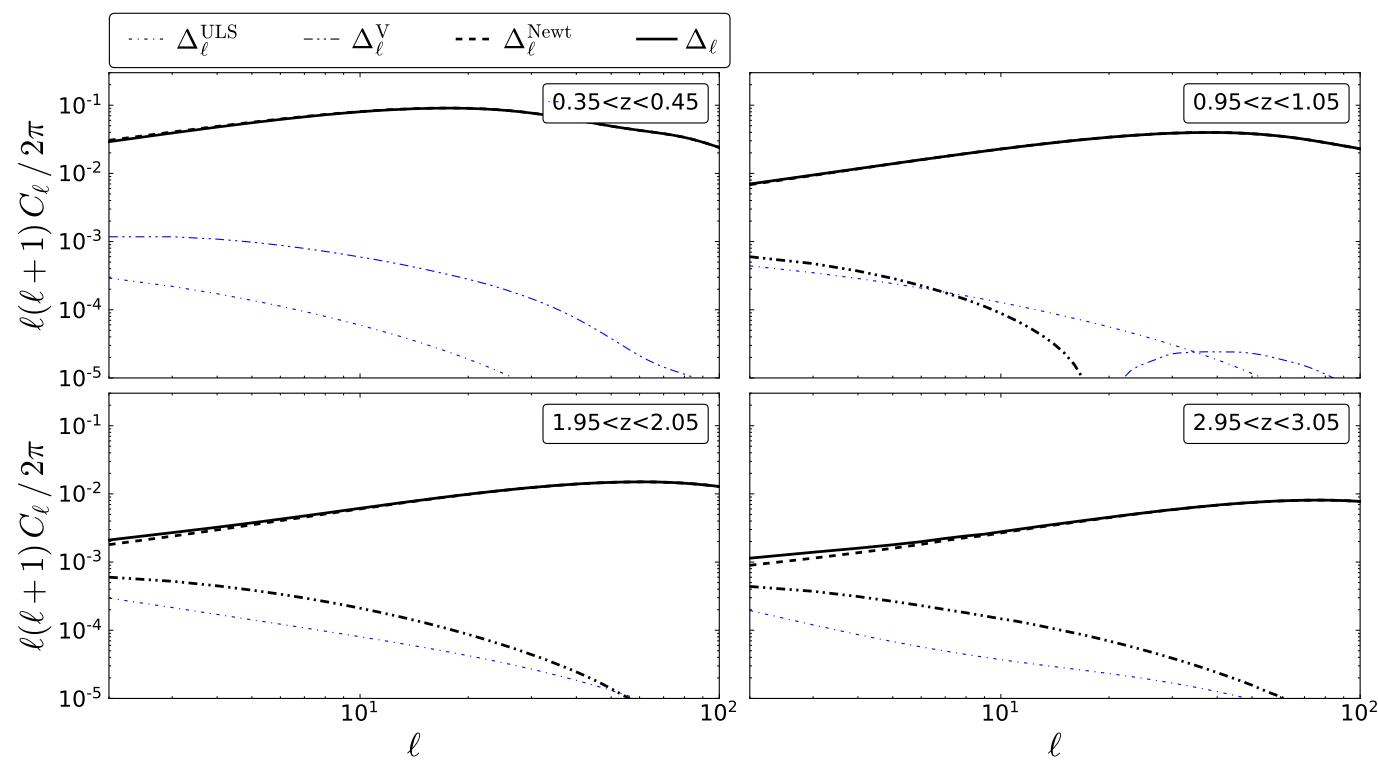

Figure 1. HI temperature contrast-ISW cross-correlation for an SKA1-MID IM survey, showing 4 of the 27 redshift bins. Different lines correspond to the contributions from ultra-large scale GR effects $\left(\Delta^{\mathrm{ULS}}\right.$, dot-dashed), from Doppler effects $\left(\Delta^{\mathrm{V}}\right.$, dot-dot-dashed), from the Newtonian approximation $\left(\Delta^{\mathrm{N}}\right.$, dashed), and from the total $(\Delta$, solid). Thin (blue) lines indicate the absolute value of a negative contribution.

Table 2. SNR for ISW detection from continuum galaxy surveys with ASKAP and SKA1-MID, using binning configurations (26) and (27). SNR is calculated using the full number count contrast $\Delta^{\mathrm{N}}+\Delta^{\mathrm{GR}}$ given by (1) (left column) and its Newtonian approximation $\Delta^{\mathrm{N}}$ given by (2) (right column). Numbers in round brackets indicate neglect of the contribution from cross-bin correlations in (14).

\begin{tabular}{ccccccccc}
\hline & \multicolumn{2}{c}{ EMU } & \multicolumn{2}{c}{ SKA1-MID Band 1 } \\
\multicolumn{2}{c}{$S_{\text {cut }}=100 \mu \mathrm{Jy}$} & \multicolumn{2}{c}{$\begin{array}{c}\text { SKA1-MID Band } 1 \\
\text { cut }\end{array}=22 \mu \mathrm{Jy}$} & \multicolumn{2}{c}{ SKA2 } \\
& $\Delta$-ISW & $\Delta^{\mathrm{N}}$-ISW & $\Delta$-ISW & $\Delta^{\mathrm{N}}$-ISW & $\Delta$-ISW & $\Delta^{\mathrm{N}}$-ISW & $\Delta$-ISW & $\Delta^{\mathrm{N}}$-ISW \\
\hline 1 bin & 5.0 & 5.0 & 4.0 & 4.0 & 5.1 & 5.1 & 5.6 & 5.6 \\
5 bins & - & - & $5.0(5.1)$ & $5.0(5.2)$ & $5.1(5.3)$ & $5.1(5.4)$ & $6.2(6.0)$ & $6.2(6.6)$ \\
\hline
\end{tabular}

cient spectroscopic information will be found (from crossmatching with $21 \mathrm{~cm}$ and optical/ infrared surveys) to construct $\Delta z=0.5$ bins up to $z=2$.

Thanks to the wide sky coverage, we find a SNR of $\sim 5$ for EMU, despite its higher flux threshold. We obtain SNR $\sim 4.0-5.0$ for the SKA1 survey with the baseline $S_{\text {cut }}=22$ $\mu \mathrm{Jy}$. For the optimistic case of $S_{\text {cut }}=10 \mu \mathrm{Jy}$, we find SNR $\sim$ 5.1. For SKA2, with a larger sky area 30,000 $\mathrm{deg}^{2}$ and lower flux threshold $1 \mu \mathrm{Jy}, \mathrm{SNR} \sim 5.6-6.2$. Similar to IM, tomography improves the SNR, although the improvement is negligible in the $10 \mu \mathrm{Jy}$ case.

Unlike IM surveys, continuum galaxy surveys are affected by lensing magnification. Figure 2 shows that the lensing contribution $\Delta^{\mathrm{L}}$-ISW (dotted line) to the full crosspower spectrum (solid line) is important at high redshift and on the large scales where the ISW signal is stronger. It is of the same order of magnitude as the $\Delta^{\mathrm{N}}$-ISW contribution (dashed line) for the highest redshift bins.

In figure 2 , we also see that the correlation between $\Delta^{\mathrm{L}}$ 
Table 3. SNR for ISW detection for an SKA2 HI galaxy survey, using binning configurations (29) and (30). SNR is calculated using the full number count contrast $\Delta^{\mathrm{N}}+\Delta^{\mathrm{GR}}$ given by (1) (left column) and its Newtonian approximation $\Delta^{\mathrm{N}}$ given by (2) (right column). Numbers in round brackets indicate neglect of the contribution from cross-bin correlations in (14).

\begin{tabular}{ccc}
\hline & \multicolumn{2}{c}{ SKA2 } \\
\hline & $\Delta$-ISW & $\Delta^{\mathrm{N}}$-ISW \\
\hline 1 bin & 3.8 & 3.7 \\
$\Delta z=0.1$ bins & $6.0(6.1)$ & $6.0(5.3)$ \\
\hline
\end{tabular}

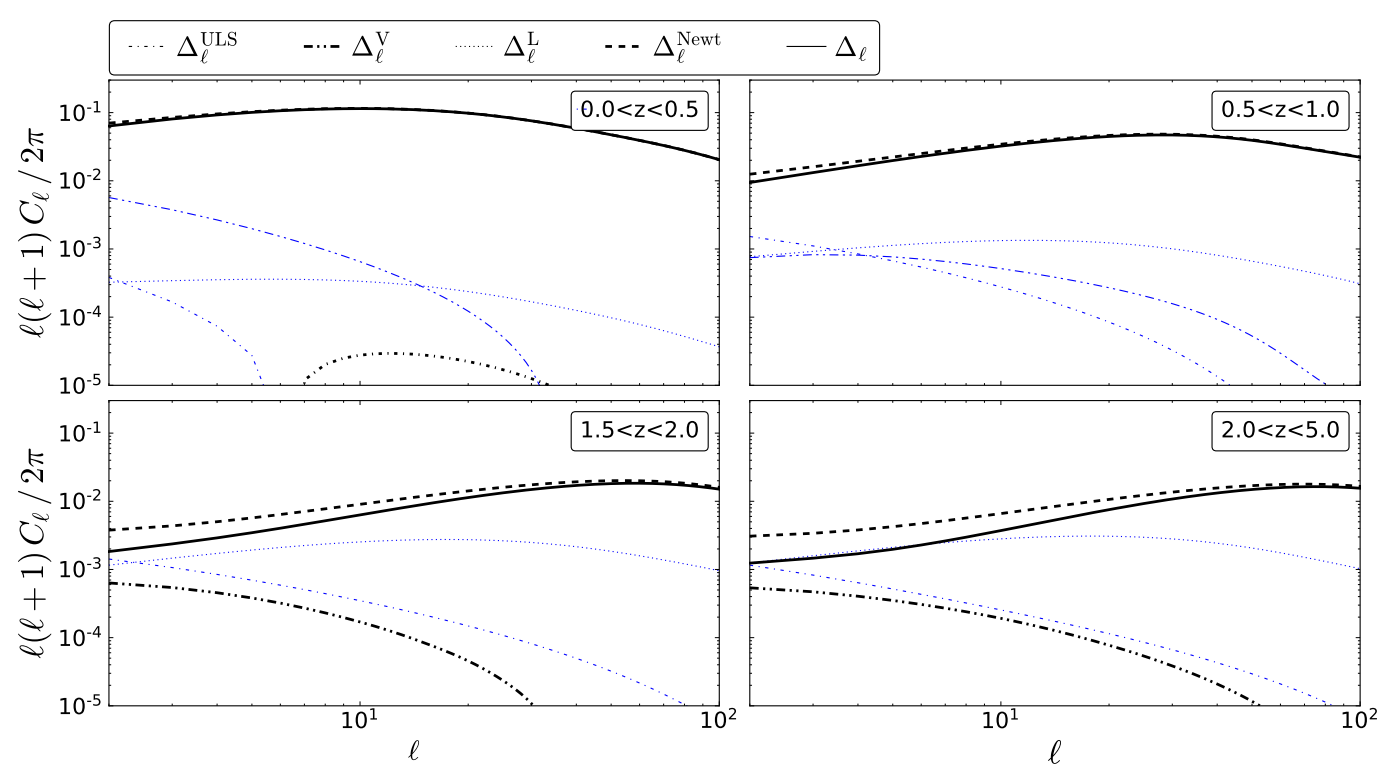

Figure 2. As in figure 1, for an SKA1 continuum survey $\left(S_{\text {cut }}=22 \mu \mathrm{Jy}\right)$, showing 4 of the 5 redshift bins. The lensing contribution (dotted), absent in figure 1 , is from $\Delta^{\mathrm{L}}$.

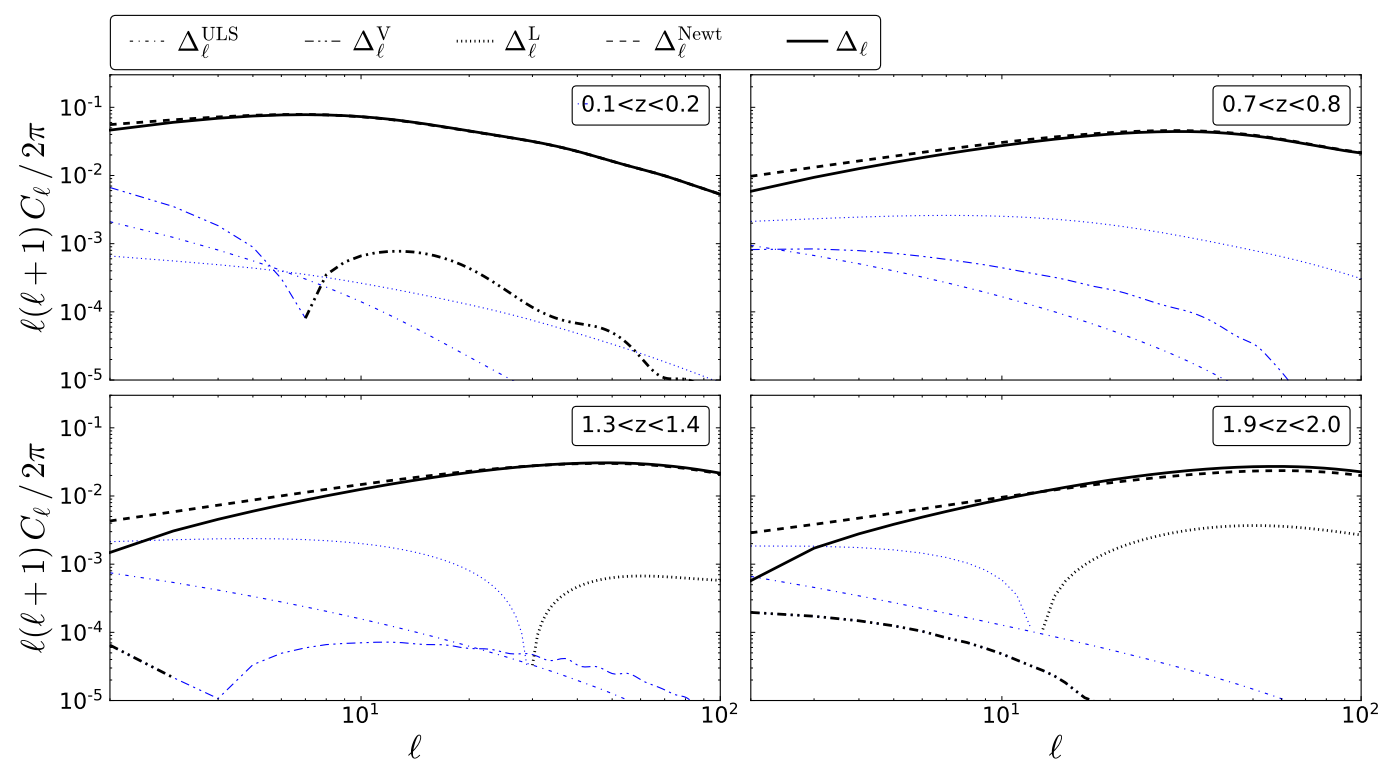

Figure 3. As in figure 2, for an SKA2 HI galaxy survey, showing 4 of the 19 redshift bins. 

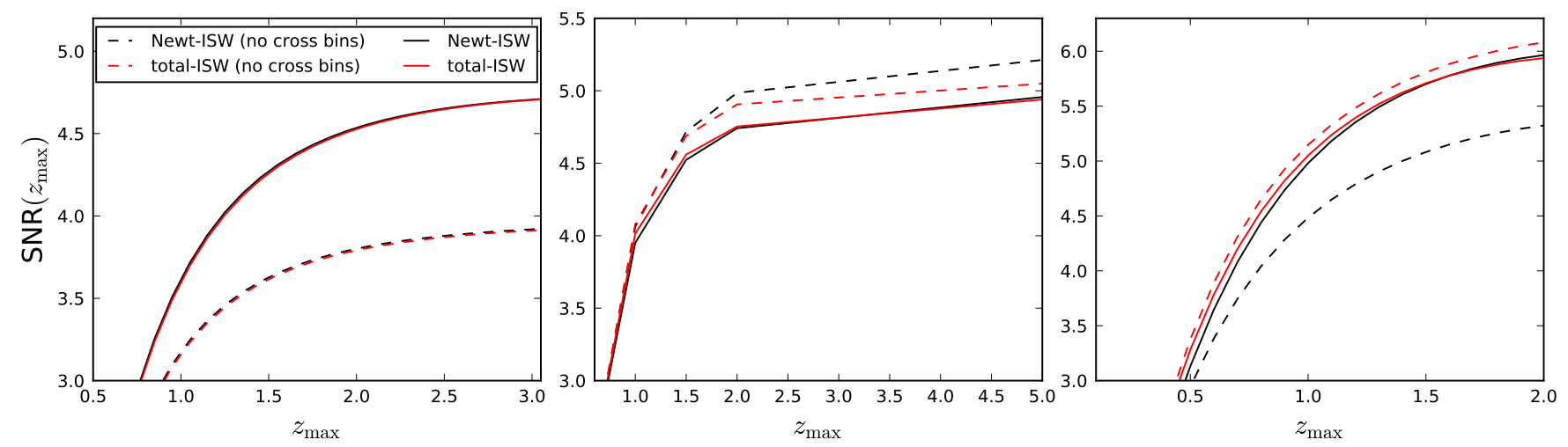

Figure 4. Cumulative SNR up to $z_{\max }$ for SKA1 IM (left panel), SKA1 continuum $\left(S_{\text {cut }}=22 \mu \mathrm{Jy}\right.$ ) (central panel), and SKA2 HI galaxy survey (right panel). Different lines correspond to the cross-correlation $\Delta^{\mathrm{N}}$-ISW (red) and $\Delta$-ISW (black), with (solid) and without (dashed) cross-bin correlations.

and ISW is always negative (thin blue lines) and it is the dominant GR correction for $z \gtrsim 1$. This lensing contribution reduces the cross-power spectrum relative to the Newtonian approximation $\Delta^{\mathrm{N}}$-ISW.

Table 2 shows the following features in the tomographic case. If we neglect the galaxy cross-bin correlations in the covariance (15) (which gives a biased SNR), then the SNR is larger in the Newtonian approximation (no lensing) than in full GR (with lensing). This means that lensing effects within each bin reduce the SNR (consistent with figure 2).

The SNR without cross-bin correlations is also larger than the SNR in the cross-bin case, with the exception of the SKA2 case. This means that correlations amongst number count contrast, RSD and lensing across different bins reduce the SNR, except for SKA2 in the full GR case, where there is an increase due to lensing. Neglecting cross-bin correlations in continuum leads to a false increase in SNR (a decrease for SKA2 in full GR) and a false excess SNR in the Newtonian approximation, due to incorrect modelling of the covariance.

Finally, table 2 also shows that when cross-bin correlations are included, the SNR in the Newtonian approximation (i.e. no lensing) differs negligibly from the SNR in the full $G R$ model (including lensing). This means that the total lensing contribution in (14), from correlations of the form

$$
\begin{aligned}
& \left\langle\Delta^{\mathrm{L}}\left(\boldsymbol{n}, z_{\mathrm{i}}\right) T^{\mathrm{ISW}}\left(\boldsymbol{n}^{\prime}\right)\right\rangle \text { (within bins) and } \\
& \left\langle\Delta^{\mathrm{L}}\left(\boldsymbol{n}, z_{\mathrm{i}}\right) \Delta\left(\boldsymbol{n}^{\prime}, z_{\mathrm{j}}\right)\right\rangle \text { (within and across bins), }
\end{aligned}
$$

nearly cancels, so that neglecting lensing does not bias the SNR appreciably.

\subsection{HI galaxy survey}

Table 3 summarises the SNR obtained for detection of the correlation of number count contrast and ISW for an SKA2 HI galaxy survey. We use two binning configurations:

$$
\begin{aligned}
& 1 z \text {-bin with edges }[0.1,2] \\
& 19 z \text {-bins with } \Delta z=0.1
\end{aligned}
$$

Figure 3 shows that the $\Delta^{\mathrm{L}}$-ISW contribution to the observed number count contrast-ISW cross-correlation is negative on the largest scales, similar to the continuum case, but can be positive at smaller scales (with $\ell<100$ ). Tomography gives a major boost to the SNR, which reaches $\sim 6$ in the full model. Table 3 shows that if cross-bin correlations are neglected, the SNR is smaller in the Newtonian approximation - which means that lensing effects within each bin increase the SNR. Without cross-bin correlations, the SNR in the full GR model is larger (6.1). This means that lensing effects across different bins reduce the SNR from its biased to true value (6.0). In other words, neglecting cross-bin correlations in SKA2 galaxy surveys leads to a false increase in SNR and a false deficit of SNR in the Newtonian approximation, due to incorrect modelling of the covariance.

Table 3 also shows that, as in the case of continuum, when cross-bin correlations are included, SNR in the Newtonian approximation (i.e. no lensing) differs negligibly from the SNR in the correct model (full GR, including lensing). It follows that correlations of the form (28) again effectively cancel.

\subsection{Summary: cumulative SNR for SKA}

The cumulative SNR as a function of $z_{\max }$ for the baseline SKA1 surveys and the SKA2 HI galaxy survey is shown in figure 4. The plots illustrate the key features identified in the previous subsections. They also show that the SNR continues to grow at redshifts where the ISW is very small.

\section{RECONSTRUCTING THE ISW SIGNAL}

We use the optimal minimum-variance $\hat{a}_{\ell \mathrm{m}}^{\mathrm{ISW}}$ estimator derived in Barreiro et al. (2009); Manzotti \& Dodelson (2014) to reconstruct the ISW signal from CMB and LSS maps (see also applications of the same estimators in Muir \& Huterer 2016; Weaverdyck et al. 2018):

$\hat{a}_{\ell \mathrm{m}}^{\mathrm{ISW}}=\sum_{i} R_{\ell}^{i} a_{\ell m}^{i}$,

where $i=1, \cdots, n$ and the reconstruction filter derived from the covariance matrix is:

$R_{\ell}^{i}=-N_{\ell}\left(D_{\ell}^{-1}\right)_{1, i}$. 

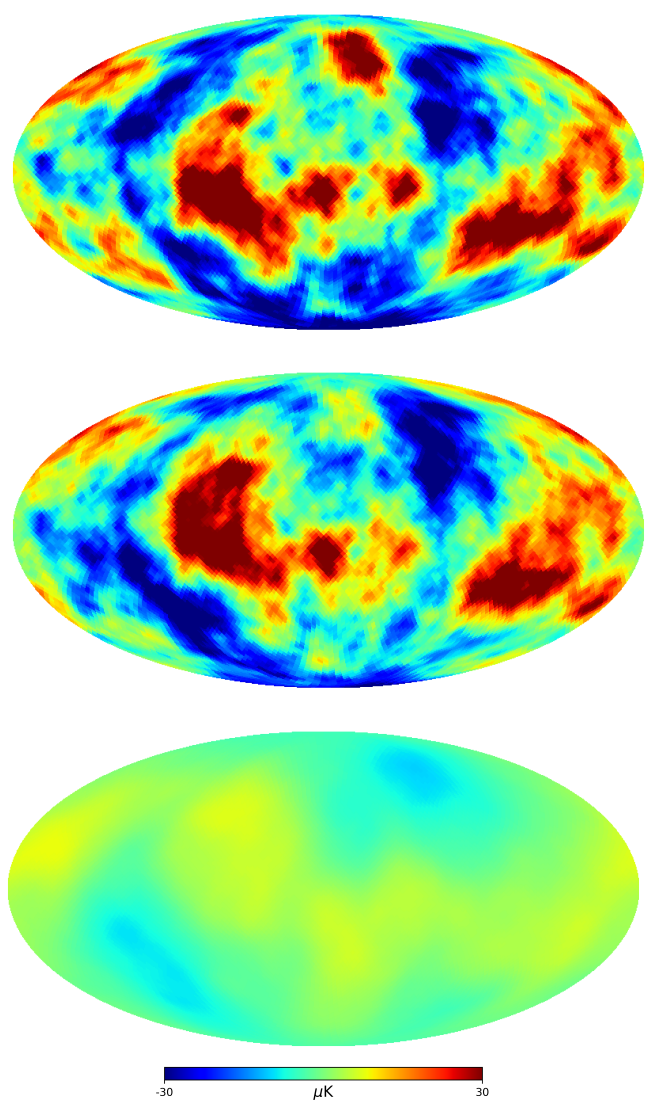

Figure 5. Example of the reconstruction procedure applied to simulation data, see (31). Top: input ISW map; centre: recovered ISW estimate using CMB temperature and SKA1 continuum $\left(S_{\text {cut }}=22 \mu \mathrm{Jy}\right)$; bottom: recovered ISW estimate using CMB temperature alone. (All maps have resolution $N_{\text {side }}=32$.)

The covariance matrix is:

$D_{\ell}=\left(\begin{array}{ccccc}C_{\ell}^{\mathrm{ISW}} & C_{\ell}^{\mathrm{ISW} \Delta_{1}} & \ldots & C_{\ell}^{\Delta_{1} \mathrm{ISW}} & C_{\ell}^{\mathrm{ISW}} \\ C_{\ell}^{\Delta_{1} \mathrm{ISW}} & \bar{C}_{\ell}^{\Delta_{1}} & \ldots & \ldots & C_{\ell}^{\Delta_{1} \mathrm{ISW}} \\ \vdots & \vdots & \ddots & \vdots & \vdots \\ C_{\ell}^{\Delta_{n} \mathrm{ISW}} & \ldots & \ldots & \bar{C}_{\ell}^{\Delta_{n}} & C_{\ell}^{\Delta_{n} \mathrm{ISW}} \\ C_{\ell}^{\mathrm{ISW}} & C_{\ell}^{\mathrm{ISW} \Delta_{1}} & \ldots & \bar{C}_{\ell}^{\Delta_{n}} & C_{\ell}^{T T}\end{array}\right)$

Here the estimated variance of the reconstruction is:

$N_{\ell}=\left[\left(D_{\ell}^{-1}\right)_{1,1}\right]^{-1}$.

We reconstruct the ISW using the CMB temperature and $n$ LSS maps.

Following Manzotti \& Dodelson (2014); Bonavera et al. (2016), we show for illustrative purposes an example of the reconstructed ISW map in figure 5 . The reconstruction using simulated data of a SKA1-MID Band 1 radio continuum survey with $S_{\text {cut }}=22 \mu$ Jy with $5 z$-bins (corresponding to a SNR 5.0) (central panel), shares a number of the visual features in common with the input ISW map (top panel). For comparison, we show in the bottom panel the ISW reconstruction obtained with the CMB temperature map alone (reducing (31) to a Wiener filter), where only a very large scale feature is captured.

\subsection{Reconstruction validation}

To quantify the accuracy of a given reconstruction, we use the correlation coefficient between the true ISW map $T^{\mathrm{ISW}}$ and the reconstructed ISW map $\hat{T}^{\mathrm{ISW}}$ :

$\rho=\frac{\left\langle T^{\mathrm{ISW}} \hat{T}^{\mathrm{ISW}}\right\rangle_{\mathrm{pix}}}{\sigma_{\mathrm{ISW}} \sigma_{\mathrm{rec}}}$,

where $\sigma_{\text {ISW }}\left(\sigma_{\text {rec }}\right)$ is the variance of the true (reconstructed) ISW map. We consider also a second statistical estimator, because the estimator (35) is insensitive to changes in the overall amplitude of the reconstructed ISW map. The reconstructed map residual is defined as:

$s=\frac{\left\langle\left(T^{\mathrm{ISW}}-\hat{T}^{\mathrm{ISW}}\right)\right\rangle_{\mathrm{pix}}^{1 / 2}}{\sigma_{\mathrm{ISW}}}$.

We calculate $\rho$ and $s$, averaged over 10,000 simulations, using the following general pipeline:

- Fiducial cosmological model is flat, with best-fit parameters: $\omega_{\mathrm{b}} \equiv \Omega_{\mathrm{b}} h^{2}=0.02218, \omega_{\mathrm{c}} \equiv \Omega_{\mathrm{c}} h^{2}=0.1205, h_{0}=$ $0.6693, \tau=0.0596, n_{\mathrm{s}}=0.9619$, and $\log \left(10^{10} A_{\mathrm{s}}\right)=3.056$, from the 2015 analysis of Planck data (Aghanim et al. 2016). LSS survey specifications are given in section 4 .

- Compute the observed auto- and cross-correlation angular power spectra (10), including the GR corrections with a modified version of CAMB_sources ${ }^{2}$ (Challinor \& Lewis 2011).

- Generate correlated Gaussian realisations of the CMB and LSS maps using HEALPix ${ }^{3}$ (Gorski et al. 2005):

$a_{\ell m}^{i}=\sum_{j=1}^{i} \xi_{j} T_{i j}$,

where $\xi$ is a complex random number with unit variance $\left\langle\xi \xi^{*}\right\rangle=1$ and zero mean $\langle\xi\rangle=0$, satisfying $\left\langle\xi_{i} \xi_{j}^{*}\right\rangle=\delta_{i j}$. The amplitudes $T_{i j}$ are generated with the following recursive expression (Giannantonio et al. 2008) to guarantee that any two maps will be correlated:

$\begin{array}{ll}T_{i j}=\left(C^{j i}-\sum_{k=1}^{j-1} T_{i k}^{2}\right)^{1 / 2} & \text { if } i=j, \\ T_{i j}=\left(T_{j j}\right)^{-1}\left(C^{j i}-\sum_{k=1}^{j-1} T_{i k} T_{j k}\right) & \text { if } i>j,\end{array}$

where the index $i$ runs over the number of maps (CMB and LSS).

- The noise for the LSS maps, i.e. shot-noise for the galaxy surveys and instrumental noise for IM, is also generated in the form of $a_{\ell m}^{\mathcal{N}}$ as a Gaussian uncorrelated map with respect to the other fields.

- Construct the covariance (33) with a set of auto- and cross-correlation angular power spectra $C_{\ell}^{\mathrm{XY}}$. As we did for the SNR estimation, we test both the impact of including the GR corrections (always included in the input maps/ simulations) and neglecting cross-bin correlations in the covariance (33).

${ }_{3}^{2}$ https://github.com/cmbant/CAMB/tree/CAMB_sources 
Table 4. Mean reconstruction quality coefficients $\langle\rho\rangle$ and $\langle s\rangle$ of ISW map reconstructions for various combinations of input maps. First column gives the case where GR corrections are included in the estimator. Second column shows results using the Newtonian approximation. Numbers in brackets indicate that cross-bin correlations are neglected.

\begin{tabular}{|c|c|c|c|c|}
\hline & \multicolumn{2}{|c|}{$\langle\rho\rangle$} & \multicolumn{2}{|c|}{$\langle s\rangle$} \\
\hline & $R_{\ell}\left(\Delta_{\ell}^{\mathrm{N}+\mathrm{GR}}\right)$ & $R_{\ell}\left(\Delta_{\ell}^{\mathrm{N}}\right)$ & $R_{\ell}\left(\Delta_{\ell}^{\mathrm{N}+\mathrm{GR}}\right)$ & $R_{\ell}\left(\Delta_{\ell}^{\mathrm{N}}\right)$ \\
\hline MeerKLASS L-Band (1 bin) & 0.38 & 0.38 & 0.93 & 0.93 \\
\hline MeerKLASS L-Band (5 bin) & $0.54(0.43)$ & $0.54(0.43)$ & $0.84(0.91)$ & $0.84(0.90)$ \\
\hline MeerKLASS UHF-Band (1 bin) & 0.56 & 0.56 & 0.83 & 0.83 \\
\hline MeerKLASS UHF-Band (11 bin) & $0.65(0.57)$ & $0.65(0.41)$ & $0.76(0.87)$ & $0.77(0.91)$ \\
\hline SKA1 - IM (1 bin) & 0.55 & 0.55 & 0.85 & 0.85 \\
\hline SKA1 - IM (27 bin) & $0.75(0.66)$ & $0.75(0.43)$ & $0.63(0.85)$ & $0.65(0.90)$ \\
\hline EMU (1 bin) & 0.63 & 0.62 & 0.78 & 0.78 \\
\hline SKA1 - continuum $22 \mu \mathrm{Jy}$ (1 bin) & 0.67 & 0.67 & 0.75 & 0.75 \\
\hline SKA1 - continuum $22 \mu \mathrm{Jy}$ ( 5 bin) & $0.87(0.78)$ & $0.87(0.76)$ & $0.53(0.65)$ & $0.49(0.65)$ \\
\hline SKA1 - continuum $10 \mu \mathrm{Jy}$ (1 bin) & 0.93 & 0.93 & 0.39 & 0.37 \\
\hline SKA1 - continuum $10 \mu \mathrm{Jy}$ (5 bin) & $0.93(0.85)$ & $0.93(0.82)$ & $0.41(0.55)$ & $0.36(0.57)$ \\
\hline SKA2 - continuum $1 \mu \mathrm{Jy}$ (1 bin) & 0.82 & 0.82 & 0.58 & 0.60 \\
\hline SKA2 - continuum $1 \mu \mathrm{Jy}$ (5 bin) & $0.90(0.77)$ & $0.88(0.84)$ & $0.43(0.64)$ & $0.54(0.61)$ \\
\hline SKA2 - HI gal (1 bin) & 0.60 & 0.60 & 0.44 & 0.49 \\
\hline SKA2 - HI gal (19 bin) & $0.90(0.67)$ & $0.88(0.53)$ & $0.44(0.75)$ & $0.49(0.85)$ \\
\hline
\end{tabular}

- Compare the reconstructed ISW signal to the true ISW map and evaluate the accuracy of the reconstruction by using (35) and (36).

Table 4 shows the quality of the ISW reconstruction for different datasets, where values $\langle\rho\rangle \rightarrow 1$ and $\langle s\rangle \rightarrow 0$ indicate more accurate reconstruction.

We consider full-sky simulations without taking into account the mask for each single map. Bonavera et al. (2016) show that the reconstruction quality is degraded by incomplete sky coverage input datasets, even when considering spectra corrected using MASTER (Hivon et al. 2002) in order to include the mode coupling in the presence of a mask.

The values of $\langle\rho\rangle$, averaged over 10,000 simulations, follow our finding for the SNR. We find always a higher value of $\langle\rho\rangle$ when tomography is performed, even in those cases, like MeerKLASS and SKA1 continuum surveys, where there is a tiny improvement in terms of SNR. Neglecting the GR terms in the filter for the reconstruction, by using a wrong covariance matrix (33), the recovered map is not properly scaled by the filter and the quality of the reconstruction is worse. However including the cross-correlation between redshift bins compensates for this degradation and we find the same values for $\langle\rho\rangle$, even when GR terms are not included in the theoretical angular power spectra used for the covariance. The inclusion of the cross-correlation leads to a better reconstruction quality, around $\sim 10-20 \%$.

Similar findings follow for the average reconstructed map residuals $\langle s\rangle$. The correlation between the galaxy number count contrast and the ISW component of the CMB is affected both in shape and amplitude at large angular scales, by the lensing convergence contribution to the number counts (see figures 2 and 3). However, this effect becomes important at high redshift $z>1.5$, which lowers the contribution to the total ISW detection - and for this reason we do not see a significant shift in $\langle s\rangle$ when the wrong covariance matrix is used.

\section{CONCLUSIONS}

In this work, we studied the feasibility of detecting the latetime ISW imprinted on the CMB temperature anisotropies by cross-correlating CMB maps with future radio maps from the SKA. Then we investigated the reconstruction of the ISW signal combining CMB with SKA surveys. We considered two of the main three cosmological probes provided by SKA in Phase 1 (Bacon et al. 2018), namely the neutral hydrogen (HI) intensity mapping survey and the radio continuum galaxy survey, together with their two precursor surveys, MeerKLASS (Santos et al. 2017) and EMU (Norris et al. 2011). We also considered the more futuristic SKA2 for radio continuum and the HI galaxy survey (the 'billion galaxy' spectroscopic survey).

We began by quantifying the theoretical signal-to-noise ratio (SNR) for ISW detection through angular cross-power spectra of CMB temperature and number count/ intensity contrast. One of the key factors to maximize the synergy between CMB and LSS maps is to have the largest overlapping sky area, also because $\operatorname{SNR} \propto \sqrt{f_{\text {sky }}}$. Future radio 
surveys promise to deliver maps of the dark matter distribution using $\mathrm{HI}$ with sky area $\sim 20,000-30,000 \mathrm{deg}^{2}$ so that the surveys are signal-dominated on the large scales relevant for the ISW. On the other hand, future optical/ infrared surveys such as DESI (Levi et al. 2013; Aghamousa et al. 2016a,b), Euclid (Laureijs et al. 2011; Amendola et al. 2016) and LSST (Abell et al. 2009; Alonso et al. 2018) have sky area $\sim 15,000 \mathrm{deg}^{2}$. The deeper redshift coverage of the radio surveys also increases the SNR, but not significantly, as shown in figure 4 . This is due to the fact that matter-temperature correlations decrease rapidly with redshift, since dark energy is subdominant at high redshift.

For the HI IM surveys, we find for MeerKLASS $1<$ SNR $<2(\sim 2.2$ combining the two Bands $)$ and SNR $\sim$ $3.7-4.7$ (higher with tomography) for SKA1 in Band 1: see table 1 . For the radio continuum galaxy surveys, we find for EMU that SNR $\sim 5$, while SNR $\sim 4.0-5.0$ (higher with tomography) for SKA1 in Band 1 and 5.6-6.2 (higher with tomography) for SKA2: see table 2. For the HI galaxy survey from SKA2 we find a SNR $~ 3.7-6.0$ (higher with tomography): see table 3 .

We tested the effect on the SNR of a tomographic approach, splitting the information of the surveys in different redshift bins. The results show that tomography does improve the SNR.

Then we considered the reconstruction of the ISW signal using a likelihood based minimum-variance estimator from CMB and LSS maps. We considered the ISW reconstruction from single radio surveys but splitting the surveys in different redshift bins, according to their baseline specifications and consistently with our SNR analysis.

We quantifed the accuracy of the reconstruction with two estimators, namely the correlation coefficient between the input and the reconstructed ISW map $\rho$, and the reconstructed map residual $s$. We calculated $\rho$ and $s$, averaging over 10,000 simulations, for all the radio surveys considered, with and without tomography. Our results for $\langle\rho\rangle$ and $\langle s\rangle$ are consistent with our finding for the SNR. Moreover, we find that tomography always leads to a higher quality of the ISW reconstruction for these two estimators.

We also studied the impact of observational effects on the radio surveys from lensing magnification, Doppler and other relativistic corrections, in altering the cross-correlation signal from CMB temperature and radio surveys. We found that the lensing effects do alter the angular cross-power spectra of CMB temperature and number count/ intensity contrast, as seen in figures $1-3$, while the other relativistic effects can be neglected. Lensing magnification can change the expected SNR up to $10-20 \%$ and degrade the ISW reconstruction when it is not modelled in the theoretical covariance used in (14) for the SNR and used in (31) for the reconstruction.

One of our main results is to show the importance of including cross-bin correlations of the LSS survey in computing the SNR. These correlations enter the covariance (15), and neglecting this contribution leads to significant bias in the SNR, as shown by the dashed curves in figure 4 and by the bracketed numbers in tables 1-3. When the cross-bin galaxy correlations are neglected, there is also a significant disagreement between the SNR in the Newtonian approximation and in the full GR model (i.e. including lensing). This is evident in figure 4 and in tables 2 and 3.
We showed that for the galaxy surveys, when cross-bin correlations are included, the SNR in the Newtonian approximation (i.e. no lensing) differs negligibly from the SNR in the correct model (full GR, including lensing). This implies that lensing contributions within bins and across different bins combine to effectively cancel [see (28)].

We conclude that SKA in Phase 1 promises a $\sim 5 \sigma$ detection of the ISW signal with $21 \mathrm{~cm}$ intensity mapping and radio continuum surveys, with a similar forecast for the precursor EMU survey, while a larger significance at $\sim 6 \sigma$ will be possible with SKA2 using the $21 \mathrm{~cm}$ galaxy redshift and radio continuum surveys. Moreover, we find that lensing and other relativistic observation effects on the number counts/ intensity angular power spectra have a small impact on the ISW detection and reconstruction. However, their impact on cosmological parameter estimation can be significant (Camera et al. 2015; Cardona et al. 2016; Lorenz et al. 2018).

There are a number of ways that one could improve the robustness and accuracy of our forecasts.

We considered the cross-correlation between the CMB and single radio surveys. Since the estimator (31) is able to combine any numbers of maps as input, it is possible to test the combined effect of all the radio surveys that will be provided from SKA for the ISW detection and reconstruction. However, this multi-tracer application to the ISW has been shown in Ballardini et al. (2019) to be effective for surveys which cover different redshifts, or for tracers with a very different redshift distribution.

The inclusion of maps of the lensing potential has been shown (Cooray 2002; Ferraro et al. 2015; Manzotti \& Dodelson 2014; Ade et al. 2016; Bonavera et al. 2016) to have the potential to improve the ISW reconstruction. This will be possible in light of future CMB experiments beyond Planck, able to provide better lensing maps in particular at the largest scales, i.e. $\ell<10$, where the correlation between ISW and lensing is largest (Manzotti \& Dodelson 2014).

Finally, we note that systematics will impact the ISW detection significances forecasted here. Estimation of these systematics is a major undertaking, since no cosmological radio survey has yet been implemented, and the 3 different radio surveys studied here will be affected by different systematics, requiring dedicated treatments. Further investigation can build on previous work on optical/IR surveys, for example Afshordi (2004); Hernandez-Monteagudo (2010); Bonavera et al. (2016); Muir \& Huterer (2016); Weaverdyck et al. (2018); Ballardini et al. (2019). In addition, systematics need to be taken into account in the reconstruction estimator to avoid biased results (Muir \& Huterer 2016; Weaverdyck et al. 2018). 


\section{Acknowledgments}

MB thanks R.B. Barreiro, C.A.P. Bengaly, L. Bonavera, S. Camera, F. Finelli, J. Fonseca, A. Manzotti, D. Molinari, M. Spinelli and C. Umiltà for fruitful discussions. MB and RM were supported by the South African Radio Astronomy Observatory, which is a facility of the National Research Foundation, an agency of the Department of Science and Technology (Grant No. 75415). MB is also supported by a Claude Leon Foundation fellowship and by ASI n.I/023/12/0 "Attivitá relative alla fase B2/C per la missione Euclid". RM is also supported by the UK Science \& Technology Facilities Council (Grant ST/N000668/1).

\section{REFERENCES}

Abell P. A., et al., 2009. (arXiv:0912.0201)

Ade P. A. R., et al., 2014, Astron. Astrophys., 571, A19

Ade P. A. R., et al., 2016, Astron. Astrophys., 594, A21

Afshordi N., 2004, Phys. Rev., D70, 083536

Afshordi N., Loh Y.-S., Strauss M. A., 2004, Phys. Rev., D69, 083524

Aghamousa A., et al., 2016b. (arXiv:1611.00036)

Aghamousa A., et al., 2016a. (arXiv:1611.00037)

Aghanim N., et al., 2016, Astron. Astrophys., 596, A107

Alonso D., Bull P., Ferreira P. G., Maartens R., Santos M., 2015, Astrophys. J., 814, 145

Alonso D., et al., 2018. (arXiv:1809.01669)

Amendola L., et al., 2016. (arXiv:1606.00180)

Bacon D. J., et al., 2018. (arXiv: 1811.02743)

Baldauf T., Seljak U., Desjacques V., McDonald P., 2012, Phys. Rev., D86, 083540

Ballardini M., 2019, Phys. Dark Univ., 23, 100245

Ballardini M., Finelli F., Maartens R., Moscardini L., 2018, JCAP, 1804, 044

Ballardini M., Paoletti D., Finelli F., Moscardini L., Sartoris B., Valenziano L., 2019, Mon. Not. Roy. Astron. Soc., 482

Barreiro R. B., Vielva P., Hernandez-Monteagudo C., MartinezGonzalez E., 2009, Radio Sci., 44, 5004

Barreiro R. B., Vielva P., Marcos-Caballero A., MartinezGonzalez E., 2013, Mon. Not. Roy. Astron. Soc., 430, 259

Battye R. A., Davies R. D., Weller J., 2004, Mon. Not. Roy. Astron. Soc., 355, 1339

Bernal J. L., Raccanelli A., Kovetz E. D., Parkinson D., Norris R. P., Danforth G., Schmitt C., 2018. (arXiv:1810.06672)

Bianchini F., Renzi A., Marinucci D., 2016, JCAP, 1611, 050

Bonavera L., Barreiro R. B., Marcos-Caballero A., Vielva P., 2016, Mon. Not. Roy. Astron. Soc., 459, 657

Boughn S., Crittenden R., 2004, Nature, 427, 45

Bruni M., Crittenden R., Koyama K., Maartens R., Pitrou C., Wands D., 2012, Phys. Rev., D85, 041301

Bull P., Ferreira P. G., Patel P., Santos M. G., 2015, Astrophys. J., 803, 21

Camera S., Santos M. G., Maartens R., 2015, Mon. Not. Roy. Astron. Soc., 448, 1035

Cardona W., Durrer R., Kunz M., Montanari F., 2016, Phys. Rev., D94, 043007

Challinor A., Lewis A., 2011, Phys. Rev., D84, 043516

Chang T.-C., Pen U.-L., Peterson J. B., McDonald P., 2008, Phys. Rev. Lett., 100, 091303

Cooray A., 2002, Phys. Rev., D65, 103510

Crittenden R. G., Turok N., 1996, Phys. Rev. Lett., 76, 575

Ferraro S., Sherwin B. D., Spergel D. N., 2015, Phys. Rev., D91, 083533

Fonseca J., Camera S., Santos M., Maartens R., 2015, Astrophys. J., 812, L22
Fonseca J., Maartens R., Santos M. G., 2018, Mon. Not. Roy. Astron. Soc., 479, 3490

Fosalba P., Gaztanaga E., 2004, Mon. Not. Roy. Astron. Soc., 350, L37

Frommert M., Ensslin T. A., 2008. (arXiv:0811.4433)

Giannantonio T., Scranton R., Crittenden R. G., Nichol R. C., Boughn S. P., Myers A. D., Richards G. T., 2008, Phys. Rev., D77, 123520

Giannantonio T., Crittenden R., Nichol R., Ross A. J., 2012, Mon. Not. Roy. Astron. Soc., 426, 2581

Gorski K. M., Hivon E., Banday A. J., Wandelt B. D., Hansen F. K., Reinecke M., Bartelman M., 2005, Astrophys. J., 622, 759

Granett B. R., Neyrinck M. C., Szapudi I., 2008, Astrophys. J., 683, L99

Hall A., Bonvin C., Challinor A., 2013, Phys. Rev., D87, 064026

Hernandez-Monteagudo C., 2010, Astron. Astrophys., 520, A101

Hivon E., Gorski K. M., Netterfield C. B., Crill B. P., Prunet S., Hansen F., 2002, Astrophys. J., 567, 2

Ho S., Hirata C., Padmanabhan N., Seljak U., Bahcall N., 2008, Phys. Rev., D78, 043519

Ilic S., Douspis M., Langer M., Penin A., Lagache G., 2011, Mon. Not. Roy. Astron. Soc., 416, 2688

Jeong D., Schmidt F., Hirata C. M., 2012, Phys. Rev., D85, 023504

Karagiannis D., Lazanu A., Liguori M., Raccanelli A., Bartolo N., Verde L., 2018, Mon. Not. Roy. Astron. Soc., 478, 1341

Knox L., 1995, Phys. Rev., D52, 4307

Kofman L., Starobinsky A. A., 1985, Sov. Astron. Lett., 11, 271

Kovetz E. D., et al., 2017. (arXiv:1709.09066)

Laureijs R., et al., 2011. (arXiv:1110.3193)

Levi M., et al., 2013. (arXiv: 1308.0847)

LoVerde M., Hui L., Gaztanaga E., 2007, Phys. Rev., D75, 043519

Lorenz C. S., Alonso D., Ferreira P. G., 2018, Phys. Rev., D97, 023537

Maartens R., Zhao G.-B., Bacon D., Koyama K., Raccanelli A., 2013, JCAP, 1302, 044

Maartens R., Abdalla F. B., Jarvis M., Santos M. G., 2015, PoS, AASKA14, 016

Maniyar A., Lagache G., Béthermin M., Ilić S., 2018

Manzotti A., Dodelson S., 2014, Phys. Rev., D90, 123009

McEwen J. D., Vielva P., Hobson M. P., Martinez-Gonzalez E., Lasenby A. N., 2007, Mon. Not. Roy. Astron. Soc., 376, 1211

Muir J., Huterer D., 2016, Phys. Rev., D94, 043503

Nolta M. R., et al., 2004, Astrophys. J., 608, 10

Norris R. P., et al., 2011, Publ. Astron. Soc. Austral., 28, 215

Papai P., Szapudi I., Granett B. R., 2011, Astrophys. J., 732, 27

Pourtsidou A., Bacon D., Crittenden R., 2017, Mon. Not. Roy. Astron. Soc., 470, 4251

Raccanelli A., et al., 2012, Mon. Not. Roy. Astron. Soc., 424, 801

Raccanelli A., et al., 2015, JCAP, 1501, 042

Raccanelli A., Montanari F., Bertacca D., Doré O., Durrer R., 2016, JCAP, 1605, 009

Renk J., Zumalacarregui M., Montanari F., 2016, JCAP, 1607, 040

Sachs R. K., Wolfe A. M., 1967, Astrophys. J., 147, 73

Santos M., et al., 2015, PoS, AASKA14, 019

Santos M. G., et al., 2017, in Proceedings, MeerKAT Science: On the Pathway to the SKA (MeerKAT2016): Stellenbosch, South Africa, May 25-27, 2016. (arXiv:1709.06099)

Sarkar T. G., Datta K. K., Bharadwaj S., 2009, JCAP, 0908, 019

Schiavon F., Finelli F., Gruppuso A., Marcos-Caballero A., Vielva P., Crittenden R. G., Barreiro R. B., Martinez-Gonzalez E., 2012, Mon. Not. Roy. Astron. Soc., 427, 3044

Shajib A. J., Wright E. L., 2016, Astrophys. J., 827, 116

Stölzner B., Cuoco A., Lesgourgues J., Bilicki M., 2018, Phys. Rev., D97, 063506 
Taburet N., Hernandez-Monteagudo C., Aghanim N., Douspis M., Sunyaev R. A., 2011, Mon. Not. Roy. Astron. Soc., 418, 2207 Vielva P., Martinez-Gonzalez E., Tucci M., 2006, Mon. Not. Roy. Astron. Soc., 365, 891

Weaverdyck N., Muir J., Huterer D., 2018, Phys. Rev., D97, 043515

Wilman R. J., et al., 2008, Mon. Not. Roy. Astron. Soc., 388, 1335

Wyithe S., Loeb A., 2008, Mon. Not. Roy. Astron. Soc., 383, 606

This paper has been typeset from a $\mathrm{T}_{\mathrm{E}} \mathrm{X} / \mathrm{L}_{\mathrm{A}} \mathrm{T}_{\mathrm{E}} \mathrm{X}$ file prepared by the author. 